DOI: $10.20472 /$ IAC.2020.053.013

\author{
KEITA NISHIO \\ Aoyama Gakuin University, Japan \\ TAKASHI KABURAGI \\ International Christian University, Japan \\ SATOSHI KUMAGAI \\ Aoyama Gakuin University, Japan \\ TOSHIYUKI MATSUMOTO \\ Aoyama Gakuin University, Japan \\ YOSUKE KURIHARA \\ Aoyama Gakuin University, Japan
}

\title{
UNCONSTRAINED CARDIAC SOUND MEASUREMENT METHOD UTILIZING A PNEUMATIC SENSING METHOD
}

\begin{abstract}
:
Diagnoses based on the cardiac sound by auscultation that require doctors to obtain cardiac sound in front of a patient is the de facto standard at medical institutions. From the viewpoint of home health management, the burden of the daily monitoring of cardiac sound could be reduced if we could obtain cardiac sounds without applying a stethoscope to the patient's body. However, prior studies of unconstrained bio-signal measurement methods mainly focused on the detection of low-frequency cardiac movements. We have also proposed an unconstrained cardiac movement measurement method utilizing a pneumatic sensing device. Hence, in this paper we aimed to evaluate whether our pneumatic method also shows sensitivity in the cardiac sound domain. In the proposed method, to measure cardiac sound without restraint, we designed a sheet-shaped device with a high-sensitivity pressure sensor, a rubber tube, an expanded polystyrene spacer, and two polyvinyl chlorides boards. One end of the tube is sealed with glue and the pressure sensor is attached to the other end. The tube with pressure sensor is installed in a groove on the spacer, which is sandwiched between two polyvinyl chlorides boards. When a person lies on the device, cardiac sound changes the pressure in the tube through the upper polyvinyl chloride boards. The pressure sensor then measures the pressure changes. The output signal from the sensor contains noise components as well as the cardiac sound. To extract the cardiac sound, the output signal from the pressure sensor is passed through an analog band-pass filter with a pass frequency of $0.008 \mathrm{~Hz}$ to $1 \mathrm{kHz}$ and a non-inverting amplifier with the gain of 2 . We evaluated our proposed method in a validity experiment involving a 24 -year-old healthy male. The subject was asked to lie on the sensing device, and a stethoscope was applied to his thoracic wall. The correlation coefficient between the frequency spectra calculated by the proposed method and phonocardiogram in the frequency band of $50 \mathrm{~Hz}$ to $1 \mathrm{kHz}$ was used as the evaluation index. A correlation coefficient was found of 0.80 , showing that the proposed unconstrained measurement method has promising sensitivity in the cardiac sound domain.
\end{abstract}

\section{Keywords:}

cardiac sound; pneumatic method; unconstrained sensing 
JEL Classification: 110 